DNA (hydroxy)methylation in T helper lymphocytes

Monticelli, Silvia

Trends in biochemical sciences

Published online: 20 February 2019

Volume 44 , n. 7 , p. $589-598$

Published version: https://doi.org/10.1016/j.tibs.2019.01.009

Postprint deposited in institutional repository RERO DOC Università della Svizzera italiana - USI 


\title{
DNA (hydroxy)methylation in T helper lymphocytes
}

\author{
Silvia Monticelli
}

Institute for Research in Biomedicine and Università della Svizzera italiana, Via Vincenzo Vela 6, CH-6500 Bellinzona, Switzerland

E-mail: silvia.monticelli@irb.usi.ch

\begin{abstract}
Upon recognition of an antigen, the differentiation of antigen-inexperienced naïve $\mathrm{T}$ lymphocytes into subsets able to effectively coordinate host defense is controlled by a network of transcription factors and regulatory molecules. In the cell nucleus, these factors act in the context of epigenetic modifications that influence DNA accessibility and ultimately gene expression. This review discusses recent findings about the role of DNA methylation (and its oxidized derivatives) in modulating differentiation and functions of $\mathrm{T}$ helper lymphocytes of the adaptive immune system.
\end{abstract}




\section{T Lymphocyte Differentiation}

Upon recognition of a pathogen or noxious agent and in the context of the appropriate extracellular cytokine milieu, $\mathrm{CD}^{+} \mathrm{T}$ helper lymphocytes can differentiate into an array of effector, memory, and regulatory subsets. $\mathrm{T}$ cell differentiation is not only essential for the host to be able to mount appropriate immune responses, but represents an exceptional model to study the regulation of dynamic and highly intertwined molecular processes such as cell activation, proliferation, differentiation, and plasticity. At a molecular level, such processes are regulated and mediated by the combinatorial activity of epigenetic (see Glossary) mechanisms, transcription factors, signaling molecules, and microRNAs to finally establish transcriptomes that are characteristic of each subset [14]. For example, differentiation towards the $\mathrm{T}_{\mathrm{H}} 1$ subset is favored by signaling mediated by the cytokine interleukin (IL)-12, and is characterized by the expression of the transcription factor T-BET and the cytokine interferon (IFN) $-\gamma$. On the other hand, $\mathrm{T}_{\mathrm{H}} 2$ differentiation occurs in the presence of IL-4 signaling, and is characterized by the expression of the transcription factor GATA-3 and high levels of IL-4 and IL-13. Other subsets include $\mathrm{T}_{\mathrm{H}} 17$ cells (characterized by the expression of IL-17 and the transcription factor RORC) and regulatory Treg cells, characterized by FOXP3 expression and immune suppressive functions $[1,2]$. The process of $\mathrm{T}$ cell lineage commitment is firmly interwoven with cell proliferation, and in vitro commitment to a specific lineage requires at least four cycles of cell division [5]. Lineage commitment entails that cells acquire not only the capacity to express lineage-specific factors, but also that they silence signature genes defining other lineages. The differentiated phenotype requires the acquisition of a certain level of stability (that is, the ability to 'remember' a given phenotype), while at the same time maintaining some flexibility to respond to the changing environment. Epigenetic mechanisms, including post-translational modifications of histone tails and DNA methylation, play critical roles in regulating all aspects of $\mathrm{T}$ cell differentiation, including the stability of the newly acquired phenotype [2]. A representative example is provided by the $C D 4$ gene, which during $\mathrm{T}$ cell development must be repressed in the $\mathrm{CD} 8^{+}$lineage of cytotoxic $\mathrm{T}$ cells and appropriately expressed in the $\mathrm{CD}^{+}$population of $\mathrm{T}$ helper lymphocytes. While $c i s$ and trans regulatory elements (enhancers and transcription factors) are crucial for the correct expression 
of the $C D 4$ gene during development, mature $\mathrm{T}$ cells are much less sensitive to the perturbation of some of these regulatory elements, having epigenetically 'stabilized' $C D 4$ expression at least in part through histone modifications [6]. Similarly, epigenetic changes occur during the process of T cell lineage commitment in the periphery, contributing to the polarization towards specific phenotypes [7]. This review will focus primarily on the role of DNA methylation and other covalent modifications of the genomic DNA, such as hydroxymethylation, in the regulation of T cell functions and will provide an overview of recent literature in the field and discuss open questions.

\section{DNA methylation and Demethylation Processes}

The diverse cellular phenotypes required by a multicellular organism arise from differences in gene modulation, with the spatio-temporal regulation of gene expression being interpreted by DNA-binding proteins such as transcription factors. Accessibility of transcription factors to regulatory regions in the genomic DNA is regulated by a number of mechanisms, including the extent of DNA methylation. The vertebrate genome is heavily methylated, with the majority of cytosines present in the CpG dinucleotide context being modified in somatic tissues [8-10]. The methyl group is deposited on cytosines by the action of DNA methyltransferase (DNMT) enzymes, including the de novo methyltransferases DNMT3A and DNMT3B [11] and the maintenance methyltransferase DNMT1. DNMT1 has a higher affinity for hemimethylated DNA and is therefore primarily responsible for the propagation of methylation during DNA replication and therefore across cell division [12]. Embryonic or early postnatal lethality in the absence of these enzymes underlines the importance of DNA methylation-related processes for mammalian development [11, 13]. Moreover, DNA methylation is frequently altered in human malignancies [14], and mutations in DNMT3B in humans are associated with a syndrome known as immunodeficiency, centromere instability, and facial anomalies (ICF) [15]. Mutations in DNMT3A have been described in a genetic syndrome characterized by overgrowth and intellectual disability [16] and in about $25 \%$ of the patients with acute myeloid leukemia [17]. 
Stable methylation occurs throughout the genome, for example at the level of the inactive $\mathrm{X}$ chromosome, parent-of-origin imprinted genes, and repetitive and transposable elements. The majority of DNA methylation patterns are therefore mostly static across differentiated tissues, but important changes at the level of key regulatory elements and gene bodies can occur throughout development and to control specific cellular processes $[12,18]$. It is worth mentioning that extensive changes in DNA methylation occur during embryogenesis, which will not however be discussed here; the reader is referred to a more comprehensive review of this topic [19]. Most of the unmethylated CpGs are found in $\mathbf{C p G}$ islands, containing primarily proximal regulatory regions for gene expression. However, distal active regulatory regions such as enhancers also display reduced levels of methylation [14] (Figure 1). DNA modifications can influence the access of transcription factors to DNA, for instance through the recruitment of methyl-CpG-binding domain (MBD) proteins that lead to a repressive chromatin state [20]. DNA methylation also directly interferes with the binding of transcription factors, with the notable examples of CTCF [21] and NRF1 [22]. Importantly, a largescale analysis of the impact of DNA methylation on the binding of 542 transcription factors revealed that cytosine methylation had a major effect on transcription factor binding, affecting it either positively (for instance in the case of homeodomain and NFAT families of proteins) or negatively (bHLH, bZIP and ETS families of transcription factors) [23]. Transcription factors that showed preferences for methylated sequences directly interacted with the 5-methyl group on cytosines, as shown by crystal structure analyses [23, 24]. While transcription factors can manifest different affinities for modified and unmodified DNA in vitro [23, 25], how they respond to dynamic levels of methylation in vivo remains to be fully understood for most biological systems [8, 23].

In contrast to an overall relatively stable global landscape of 5-methylcytosine (5mC), DNA methylation can be instead highly dynamic at the level of specific regulatory regions, and linked to cell type-specific enhancer activity: active regulatory regions can be indeed identified from DNA methylation data [26]. Remarkably, $5 \mathrm{mC}$ is also present within the gene bodies of actively transcribed genes $[9,27,28]$; gene body methylation of actively transcribed genes correlates with the specific binding of DNMT3B [27] and suggests that the presence of the methyl mark in this location is not 
sufficient by itself to block transcription elongation, while transcription initiation is hindered by methylation of proximal promoters $[8,29]$. Indeed, the functional role of gene body methylation of highly expressed genes might be to prevent aberrant events of cryptic intragenic transcription initiation [28].

The comprehensive characterization of the dynamics of DNA modifications is essential for a better understanding of the concept of epigenetic memory [30]. Removal of DNA methylation can be achieved through passive dilution of the methyl mark due to DNA replication during cell division, or through oxidation of the methyl group mediated by enzymes of the TET (ten-eleven translocation) family of dioxygenases (TET1, 2, 3). Using $5 \mathrm{mC}$ as a substrate, these enzymes mediate the formation of 5-hydroxymethylcytosine ( $5 \mathrm{hmC}$ ), followed either by passive dilution or iterative oxidation to 5formylcytosine $(5 \mathrm{fC})$ and 5-carboxylcytosine $(5 \mathrm{caC})$ followed by base excision repair $[19,31]$. Methods used to study the distribution of $5 \mathrm{mC}$ and $5 \mathrm{hmC}$ in the genome are summarized in Box $\mathbf{1}$. Despite their relatively recent discovery as enzymes able to oxidize $5 \mathrm{mC}$ [32], a flurry of studies demonstrated how TET enzymes can influence a great variety of processes, including differentiation and functions of immune cells [33]. For example, mutations in the TET2 gene in humans are associated with myelodysplastic syndromes and abnormal expansion of immature myeloid cells [34], and deletion of Tet genes in the B and T lymphocyte lineages in the mouse led to severe abnormalities (reviewed in [33]). Moreover, loss of Tet 2 and Tet 3 in mice induced a highly aggressive form of myeloid leukemia with high penetrance, although, despite moderate hypermethylation observed in the absence of $\operatorname{Tet} 2 / \mathrm{Tet} 3$, transcriptional changes observed in the transformed cells did not strongly correlate with changes in DNA methylation [35]. These results highlight the possibility that $5 \mathrm{hmC}$ does not act simply as an intermediate of DNA demethylation but also as an epigenetic mark in its own right. Indeed, many chromatin regulators and transcription factors able to preferentially bind $5 \mathrm{hmC}$, as compared to unmodified cytosines or $5 \mathrm{mC}$, have been identified using a mass spectrometry approach [25]. It is also possible that TET enzymes may have functions that are independent from their catalytic activity, as discussed below. 


\section{DNA (Hydroxy)methylation-Mediated Regulation of T Lymphocyte}

\section{Differentiation and Functions}

$\mathrm{T}$ cell development and differentiation are processes that cannot be separated from lymphocyte proliferation, and that are closely connected with the dynamic loss and acquisition of DNA methylation at specific regulatory regions [36]. Most results about a role of DNMT enzymes and DNA methylation in T lymphocytes have been extensively reviewed $[2,37]$ and will be briefly summarized here. The conditional deletion of the Dnmt 1 gene early in T lymphocyte development using LckCre revealed the importance of this maintenance DNA methyltransferase in the appropriate survival and lineage specification of thymocytes [38]. Deletion of the same gene at a later stage of T cell development using $C D 4 C r e$ led to an increased ability of naïve T cells to produce cytokines [38]. Similarly, loss of Dnmt3a in CD4 $4^{+} \mathrm{T}$ lymphocytes resulted in increased and dysregulated cytokine expression [39]. These results highlighted a role for DNA methylation not only in the regulation of cytokine expression, but also in the stabilization of $\mathrm{T}$ cell phenotypes, since differentiated $\mathrm{T}_{\mathrm{H}} 2$ cells lacking Dnmt3a could easily induce IFN- $\gamma$ production once placed in $\mathrm{T}_{\mathrm{H}} 1$ conditions, while wild-type cells remained much more stable in their lineage decision [39]. In terms of DNA methylation dynamics, key cis-regulatory regions (enhancers, conserved noncoding sequences (CNSs)) undergo processes of demethylation during $\mathrm{T}$ cell differentiation and polarization. For instance, a number of CNS regions are involved in regulating Ifng expression in the mouse, and several of them become variably demethylated upon differentiation of naïve T cells towards the IFN- $\gamma$-producing subset of $\mathrm{T}_{\mathrm{H}} 1$ cells, but remain methylated in IL-4-producing $\mathrm{T}_{\mathrm{H}} 2$ cells [40]. In general, differentiation of $\mathrm{T}$ cells from naïve to central memory, effector memory, and terminally differentiated effector cells correlates with a progressive reduction in genomic DNA methylation, as shown for primary human $\mathrm{CD}^{+} \mathrm{T}$ lymphocytes, with more limited variation occurring instead upon acute activation of $\mathrm{T}$ cells

\section{$[41,42]$ (Figure 2).}

In terms of $5 \mathrm{hmC}$ dynamics, during $\mathrm{T}$ cell development and differentiation in the mouse, $5 \mathrm{hmC}$ becomes enriched at regulatory regions associated with active cell-type specific enhancers, and in the different $T$ cell lineages $\left(T_{H} 1, T_{H} 2\right.$, and $\left.T_{H} 17\right)$ it is strongly associated with the regulatory regions of 
the lineage-defining cytokines IFN- $\gamma$, IL-4, and IL-17 [43, 44]. Similarly, during processes of activation and differentiation, primary human naïve $\mathrm{T}$ lymphocytes show a global loss of genomic $5 \mathrm{hmC}$, that is instead acquired at selected loci that become active only in the differentiated lineage [45] (Figure 2). As for the role of TET enzymes in T cell development, differentiation, and function, various studies addressed the effect of deleting at least one TET family member in T lymphocytes, although it became quickly clear that there is considerable redundancy among these enzymes and often a significant effect can be observed only upon deletion of more than one protein. Nevertheless, most studies point towards a role for TET enzymes in regulating at least some aspects of $\mathrm{T}$ cell development, resulting in a peripheral phenotype that can be more or less severe, depending on the system under investigation. For instance, germline deletion of mouse Tet 2 did not dramatically impact lymphocyte development, probably because of compensatory effects mediated by other TET proteins [46]. However, deletion of Tet2 in lymphoid progenitors led to a reduction in the expression of the signature cytokines IFN- $\gamma$ and IL-17A in the $\mathrm{T}_{\mathrm{H}} 1$ and $\mathrm{T}_{\mathrm{H}} 17$ subsets, respectively [43]. In CD8 ${ }^{+}$ cytotoxic $\mathrm{T}$ lymphocytes, the loss of Tet 2 promoted the acquisition of a memory phenotype, resulting in the increased ability of mice lacking Tet 2 to control pathogenic infection upon rechallenge in a model of acute viral infection [47]. Deletion of both Tet 2 and Tet3 in T cells led to uncontrolled proliferation of a normally small population of lipid-recognizing invariant natural killer (NK)T cells, leading to a lethal lymphoproliferative disease [48], as well as to impaired Treg functions mediated by unstable expression of the master transcription factor FOXP3 [49]. Specifically, TET activity was shown to be important during Treg cell development to ensure adequate demethylation of CNS1 and CNS2, key cis-regulatory elements for appropriate expression of the Foxp3 gene, located upstream of the first coding exon of Foxp3. As a results, loss of Tet 2 and Tet 3 during Treg cell development compromised FOXP3 expression and Tregs suppressive capacities [49]. Interestingly, such effect on the regulation of Foxp 3 expression could only be observed upon deletion of both Tet 2 and Tet 3 , pointing towards redundant functions of the different TET proteins in regulating differentiation and function of Tregs and other T cell subsets. Similarly, deletion of Tet 1 and Tet 2 determined hypermethylation of the Foxp3 locus, impairing T cell differentiation and function [50]. While it 
appears that the different TET family members are highly redundant in modulating Treg cell biology, whether there is also a family member-specific component to this regulation remains to be fully understood. A triple deletion of all TET proteins in Treg cells may be required to resolve this issue.

While most observations discussed so far were derived from mouse models, the recent emergence of advanced genome editing techniques (Box 2 ) allowed the efficient modification of the genome and epigenome even in primary $\mathrm{T}$ cells from human donors [51-53], opening new venues of direct investigation of these cells essential for productive immune responses in humans. However, despite such great advances in the field and the potential for these tools to open new possibilities in therapeutic applications using T cells [52], there are still some limitations that need to be overcome. Most importantly, methods of primary $\mathrm{T}$ cell editing still require $\mathrm{T}$ cell activation and extensive in vitro culture, hindering for example our ability to study naïve (non-antigen experienced) $\mathrm{T}$ lymphocytes.

\section{5hmC: Intermediate of DNA Demethylation or Stable Epigenetic Mark?}

The $5 \mathrm{hmC}$ modification is enriched in gene bodies of highly expressed genes and at poised and cell type-specific active enhancers [44]. While the functional role of $5 \mathrm{hmC}$ in gene bodies remains to be understood, regulatory regions may undergo TET-mediated DNA demethylation processes in order to maintain their accessibility to transcription factors. Some examples in this direction exist in the literature; for instance, impaired demethylation of C/EBP (CCAAT/ enhancer-binding protein)dependent enhancers determined a complex phenotype in mice that phenocopied at least in part Cebp deficiency [54]. However, the relative contribution of passive and active mechanisms of DNA methylation turnover at active enhancers remains to be determined for most biological systems. For instance, interference between transcription factor binding and the DNA methylation enzymes may also lead to an indirect loss of methylation [8]. A further possibility that cannot be disregarded is that the $5 \mathrm{hmC}$ enrichment at the level of enhancers does not simply represent an intermediate of demethylation, but rather a distinct process of enhancer activation, for example through the 
recruitment of factors yet to be identified [55]. An observation potentially in favor of this latter possibility is the fact that single-nucleotide resolution mapping of $5 \mathrm{hmC}$ and its oxidized products $5 \mathrm{fC}$ and $5 \mathrm{caC}$ in murine embryonic stem (ES) cells showed the expected enrichment at distal regulatory elements, but very little positional overlap between the three oxidized forms of $5 \mathrm{mC}$, with the majority of $5 \mathrm{hmC}$ stably marking the genome without being efficiently converted to $5 \mathrm{fC} / 5 \mathrm{caC}$ [56]. The authors further suggested that TET enzymes might preferentially stall at the $5 \mathrm{hmC}$ step, although they exhibit higher processivity at chromatin regions characterized by high accessibility [56]. Accordingly, $5 \mathrm{hmC}$ was shown to be a stable modification in the genomic DNA, as determined by isotope labeling in mammalian cells and tissues [57].

One intriguing possibility is that $5 \mathrm{hmC}$ may be important to somehow mark enhancers that have to remain in an accessible state, regardless of whether or not the associated gene is being transcribed in that particular moment. For instance, the enhancers becoming active during brain development are specifically enriched with $5 \mathrm{hmC}$ prior to their activation, with the establishment of adult $5 \mathrm{hmC}$ patterns for cell type-specific genes occurring in utero in the immature fetal brain [58]. These observations suggest that TET enzymes may be important during development and differentiation to establish a silent state in tissue-specific enhancers that is however poised for activation at later stages or in response to appropriate extracellular cues. Similarly, marking of enhancers with $5 \mathrm{hmC}$ in ES cells was shown to be required for subsequent cell-type restricted enhancer activation in differentiated cells such as macrophages [59]. These 'premarked' macrophage enhancers were not functional in ES cells, but maintained an open chromatin configuration, likely to be important in actively dividing ES cells to maintain a 'memory' of differentiation potential. Finally, TET protein activity was required in human ES cells to maintain the promoter of the $P A X 6$ gene in a demethylated state, thereby allowing gene activation upon differentiation towards the neuronal lineage [60]. As for immune cells, a careful look into the dynamics of $5 \mathrm{hmC}$ during $\mathrm{T}$ cell development in the thymus may provide some hints as to whether $5 \mathrm{hmC}$ might indeed play a role in pre-marking naïve $\mathrm{T}$ cell enhancers that will become fully functional only upon antigen recognition and activation. Briefly, during T cell development the most immature thymocytes are defined as double-negative (DN) cells, still lacking expression of the T 
cell receptor (TCR) and of the surface markers CD4 and CD8. Productive rearrangement of the TCR $\beta$ chain leads to the transition towards a double-positive (DP) stage, characterized by the expression of both CD4 and CD8. Positive selection of cells able to recognize MHC class I or class II leads to the development of thymocytes that are single-positive (SP) for either CD8 or CD4, respectively. Interestingly, the highest levels of genomic $5 \mathrm{hmC}$ can be identified at the SP stage, similarly to peripheral naïve T cells (Vincenzetti and Monticelli, unpublished observation). Since 5hmC becomes enriched at thymus-specific enhancers during the lineage commitment from DP to the SP (CD4 or CD8) stages [44], these data suggest that $5 \mathrm{hmC}$ may have indeed a role in 'protecting' from silencing specific enhancers that will be required later during an immune response, and that $5 \mathrm{hmC}$ deposition is somehow already instructed in the thymus during $\mathrm{T}$ cell development. Consistent with this possibility, a recent study showed that in murine ES cells TET1 might limit DNA methylation at regulatory regions at least in part by protecting the target region from the action of DNMT3A [61]. The relatively elevated levels of $5 \mathrm{hmC}$ at transcribed genes and active enhancers may also contribute to protect these regions from mutational damage $[62,63]$. Indeed, compared to $5 \mathrm{mC}$ that can frequently undergo $\mathrm{C}>\mathrm{T}$ mutations, $5 \mathrm{hmC}$ was found to mutate less frequently, potentially contributing to the maintenance of cell functionality especially in resting cells with limited renewal potential, such as neurons but also primary human naïve T lymphocytes [62, 63].

A further aspect that needs to be considered and that remains mechanistically somewhat unexplored is that of the relative contribution of DNA demethylation-dependent and -independent functions of DNA-modifying enzymes. For instance, we found that TET2 was able to regulate mast cell differentiation and proliferation through activities that were both catalytic-dependent and independent [64]. Although the mechanisms behind such catalytic-independent functions of TET proteins remain to be fully explored, they are likely to involve interactions with other co-regulators. For example, TET1 was shown to contribute to transcriptional repression, and its incorporation into the SIN3A corepressor complex determined transcriptional effects that were independent of the conversion of $5 \mathrm{mC}$ to $5 \mathrm{hmC}$ [65]. Moreover, in myeloid cells TET2 was shown to repress transcription of the $I l 6$ gene via recruitment of HDAC2 and histone deacetylation [66]. The proportion 
of genes that can be regulated by $5 \mathrm{hmC}$-independent, yet TET-dependent mechanisms remains however to be investigated for most biological systems.

\section{Concluding Remarks}

Our understanding on how the dynamics of DNA methylation regulate gene expression is still incomplete (see Outstanding Questions), and whether mechanisms of DNA demethylation in the different biological systems are predominantly active (TET-dependent) or passive (DNA replicationdependent) remains for the most part to be understood. Indeed, one possibility is that $5 \mathrm{hmC}$ may act primarily as an epigenetic mark in its own right, and that erasure of $5 \mathrm{mC}$ (or $5 \mathrm{hmC}$ ) from the genome remains instead a predominantly passive, replication-dependent mechanism. In terms of correlation with disease, mutations in TET2 and DNMT3A can predispose to, but are insufficient for, the development of leukemia [67]. On the other hand, loss of Tet 2 and Tet 3 in the mouse led to aggressive myeloid leukemia, establishing a causal link between TET loss-of-function and tumor development that was associated, at least in part, to defects in DNA damage responses [35]. Moreover, the combination of loss-of-function mutations in TET2 with the G17V mutation in the Rho GTPase RhoA was shown to induce abnormal $\mathrm{T}$ cell proliferation and differentiation, resembling at least a subset of patients with peripheral $\mathrm{T}$ cell lymphoma [68] and indicating that these mutations may somehow cooperate to induce the aberrant $\mathrm{T}$ cell features observed in this type of disease. Finally, altered DNA methylation is frequently observed in immune-related disorders, including autoimmune diseases such as systemic lupus erythematosus [37, 69]. Further mechanistic studies will undoubtedly shed light on the role of TET proteins and $5 \mathrm{hmC}$ in normal immune responses and during disease. 


\section{Box 1}

\section{Methods to detect DNA modifications}

DNA modifications can be detected using a variety of techniques [70]. The gold standard method to analyze DNA methylation is bisulfite treatment, which converts unmethylated cytosines to uracils through deamination, while methylated cytosines are protected. However, whole-genome bisulfite sequencing is costly, and other methods are commonly used to enrich methylated regions in the genome and therefore reduce the amount of DNA that has to be sequenced. One of these methods is reduced representation bisulfite sequencing (RRBS), in which a methylation-insensitive restriction enzyme such as MspI (which cuts 5'-CCGG sequences) is used to fractionate CpG-rich DNA, followed by bisulfite conversion. This method provides an enrichment of genomic regions with relatively high $\mathrm{CpG}$ density, covering only a portion of the total CpGs present in the human genome, primarily located in $\mathrm{CpG}$ islands and promoter regions [70]. However, bisulfite treatment and RRBS do not distinguish between $5 \mathrm{mC}$ and its oxidized modifications. Enrichment of the modified DNA using antibody specific against $5 \mathrm{mC}$ or $5 \mathrm{hmC}$ followed by sequencing has therefore become a common method for profiling DNA modifications genome-wide. Such methods of methylated or hydoxymethylated DNA immunoprecipitation (MeDIP or hMeDIP) followed by sequencing are convenient and common, although one should be aware of possible shortcomings. First, they are not single-nucleotide resolution methods, since they allow the identification of modified regions, but with no information about specific nucleotides. Second, like all antibody-based methods, the utilized antibodies should be carefully controlled for specificity and validated independently, in order to ensure correct interpretations of the data [71]. Techniques that can discriminate $5 \mathrm{hmC}$ from other forms of cytosine at a single-base resolution include TET-assisted bisulfite sequencing (TAB-seq) [72] and oxidative bisulfite sequencing (oxBS-seq) [73]. In these methods $5 \mathrm{hmC}$ is either protected (TAB-seq) or specifically modified (oxBS-seq) prior to bisulfite treatment and sequencing. By comparing the sequencing data from the same DNA treated and untreated, it is then possible to distinguish the different cytosine modifications from one another [74]. More recently, a new enzymatic method called APOBEC-coupled epigenetic sequencing (ACE-seq) was developed to map 
5hmC at a single-base resolution [75]. This method uses an AID/ APOBEC family DNA deaminase and has the advantage of not relying on bisulfite treatment, which often poses important technical challenges such as the degradation of $>90 \%$ of the genomic DNA. Importantly, single-cell methods for DNA methylation profiling have also recently been developed, which will undoubtedly provide important answers about DNA methylation dynamics especially regarding heterogeneous systems. I refer the reader to a more extensive review about these methods [76].

\section{Box 2}

\section{Epigenome editing}

Establishing whether a causative relationship exists between a certain DNA modification and changes in gene expression is often challenging. Newly developed approaches of 'epigenome editing' have now the potential to provide more conclusive functional answers on the role of $5 \mathrm{mC}$ and $5 \mathrm{hmC}$ in transcriptional regulation [77]. Site-specific epigenome editing is made possible by the fusion of DNA-modifying proteins (DNMT or TET enzymes) to specific DNA-binding domains or a catalytically inactive variant of the Cas9 nuclease (dCas9). The CRISPR-Cas9 system is particularly interesting because it can be exploited to target an enzymatic activity to a precise genomic location by specifying the sequence of the guide RNA (gRNA). For example, expression of a fusion protein between the catalytic domain of TET1 or DNMT3A and dCas9 led to the activation or silencing of methylated or unmethylated promoters, respectively $[51,78,79]$, highlighting the feasibility and potential of this approach. Two important issues to consider when attempting site-specific epigenome editing include the stability of the induced modification, and the specificity of the fusion protein. While several studies showed clear specificity of the CRISPR-Cas9 system fused to epigenetic modifiers, it is also challenging to trace the global activity of the Cas9-DNA methyltransferase fusion protein in the context of a genome that is already mostly methylated. A recent study addressed the question of epigenome-editing specificity by introducing the Cas9-methyltransferase fusion protein in methylation-depleted ES cells [80]. Unexpectedly, the authors found broad and ubiquitous DNA 
methyltransferase activity of the fusion protein, regardless of the gRNA sequence, highlighting how the possibility of widespread off-target activity should be carefully assessed in all experiments attempting site-specific epigenome editing. Nevertheless, DNA methylation editing tools show undoubtedly great potential for a variety of purposes, including clinical applications. For example, a dCas9-TET1 fusion protein was effective in reversing the aberrant DNA methylation of CGG repeats in the proximity of the FMRI gene, a process that is usually observed in fragile X syndrome (FXS) patients [81]. Specific DNA demethylation of these regions led to the reacquisition of FMR1 expression and rescued neuron functions, at least in vitro, highlighting the potential for epigenome editing in future applications.

\section{Glossary}

- Epigenetics: most commonly refers to a layer of additional information beyond the one encoded in the sequence of genomic DNA. The reader is referred to an excellent commentary on the definition of this term that is at times used ambiguously [82].

- CpG islands: CpG-rich regions of DNA often associated with gene promoters.

- Hydroxymethylation: In this context it refers to the process of oxidation of 5-methylcytosine to 5-hydroxymethylcytosine in the DNA, mediated by TET enzymes.

- Hemimethylated DNA: DNA that is methylated only on one of the two complementary strands of the double helix, for example during DNA replication. 


\section{Acknowledgments}

I would like to thank all members of my laboratory for constructive discussion, Cristina Leoni for providing a template for Figure 1 and Dior Baumjohann for help with Figure 2. Work in the laboratory on this topic is supported by the Swiss National Science Foundation (grant number 156875 and 175569 to S.M.). 


\section{References}

1. Kanno, Y. et al. (2012) Transcriptional and epigenetic control of T helper cell specification: molecular mechanisms underlying commitment and plasticity. Annu Rev Immunol 30, 707-31.

2. Wilson, C.B. et al. (2009) Epigenetic control of T-helper-cell differentiation. Nat Rev Immunol 9 (2), 91-105.

3. Sallusto, F. and Monticelli, S. (2013) The many faces of CD4 T cells: roles in immunity and disease. Semin Immunol 25 (4), 249-51.

4. Monticelli, S. (2013) MicroRNAs in T helper cell differentiation and plasticity. Semin Immunol 25 (4), 291-8.

5. Grogan, J.L. et al. (2001) Early transcription and silencing of cytokine genes underlie polarization of T helper cell subsets. Immunity 14 (3), 205-15.

6. Issuree, P.D. et al. (2017) Heritable Gene Regulation in the CD4:CD8 T Cell Lineage Choice. Front Immunol 8, 291.

7. Rothenberg, E.V. and Zhang, J.A. (2012) T-cell identity and epigenetic memory. Curr Top Microbiol Immunol 356, 117-43.

8. Baubec, T. and Schubeler, D. (2014) Genomic patterns and context specific interpretation of DNA methylation. Curr Opin Genet Dev 25, 85-92.

9. Lister, R. et al. (2009) Human DNA methylomes at base resolution show widespread epigenomic differences. Nature 462 (7271), 315-22.

10. Stadler, M.B. et al. (2011) DNA-binding factors shape the mouse methylome at distal regulatory regions. Nature 480 (7378), 490-5.

11. Okano, M. et al. (1999) DNA methyltransferases Dnmt3a and Dnmt3b are essential for de novo methylation and mammalian development. Cell 99 (3), 247-57.

12. Bird, A. (2002) DNA methylation patterns and epigenetic memory. Genes Dev 16 (1), 6-21.

13. Li, E. et al. (1992) Targeted mutation of the DNA methyltransferase gene results in embryonic lethality. Cell 69 (6), 915-26.

14. Yang, L. et al. (2015) DNMT3A in haematological malignancies. Nat Rev Cancer 15 (3), 152-65.

15. Xu, G.L. et al. (1999) Chromosome instability and immunodeficiency syndrome caused by mutations in a DNA methyltransferase gene. Nature 402 (6758), 187-91.

16. Tatton-Brown, K. et al. (2014) Mutations in the DNA methyltransferase gene DNMT3A cause an overgrowth syndrome with intellectual disability. Nat Genet 46 (4), 385-8.

17. Ley, T.J. et al. (2010) DNMT3A mutations in acute myeloid leukemia. N Engl J Med 363 (25), 2424-33. 
18. Smith, Z.D. and Meissner, A. (2013) DNA methylation: roles in mammalian development. Nat Rev Genet 14 (3), 204-20.

19. Wu, X. and Zhang, Y. (2017) TET-mediated active DNA demethylation: mechanism, function and beyond. Nat Rev Genet 18 (9), 517-534.

20. Schubeler, D. (2015) ESCI award lecture: regulation, function and biomarker potential of DNA methylation. Eur J Clin Invest 45 (3), 288-93.

21. Bell, A.C. and Felsenfeld, G. (2000) Methylation of a CTCF-dependent boundary controls imprinted expression of the Igf2 gene. Nature 405 (6785), 482-5.

22. Domcke, S. et al. (2015) Competition between DNA methylation and transcription factors determines binding of NRF1. Nature 528 (7583), 575-9.

23. Yin, Y. et al. (2017) Impact of cytosine methylation on DNA binding specificities of human transcription factors. Science 356 (6337).

24. Liu, Y. et al. (2013) A common mode of recognition for methylated CpG. Trends Biochem Sci 38 (4), 177-83.

25. Spruijt, C.G. et al. (2013) Dynamic readers for 5-(hydroxy)methylcytosine and its oxidized derivatives. Cell 152 (5), 1146-59.

26. Burger, L. et al. (2013) Identification of active regulatory regions from DNA methylation data. Nucleic Acids Res 41 (16), e155.

27. Baubec, T. et al. (2015) Genomic profiling of DNA methyltransferases reveals a role for DNMT3B in genic methylation. Nature 520 (7546), 243-7.

28. Neri, F. et al. (2017) Intragenic DNA methylation prevents spurious transcription initiation. Nature 543 (7643), 72-77.

29. Appanah, R. et al. (2007) An unmethylated 3' promoter-proximal region is required for efficient transcription initiation. PLoS Genet 3 (2), e27.

30. Monticelli, S. and Natoli, G. (2013) Short-term memory of danger signals and environmental stimuli in immune cells. Nat Immunol 14 (8), 777-84.

31. Pastor, W.A. et al. (2013) TETonic shift: biological roles of TET proteins in DNA demethylation and transcription. Nat Rev Mol Cell Biol 14 (6), 341-56.

32. Tahiliani, M. et al. (2009) Conversion of 5-methylcytosine to 5-hydroxymethylcytosine in mammalian DNA by MLL partner TET1. Science 324 (5929), 930-5.

33. Tsagaratou, A. et al. (2017) TET Methylcytosine Oxidases in T Cell and B Cell Development and Function. Front Immunol 8, 220.

34. Ko, M. et al. (2010) Impaired hydroxylation of 5-methylcytosine in myeloid cancers with mutant TET2. Nature 468 (7325), 839-43.

35. An, J. et al. (2015) Acute loss of TET function results in aggressive myeloid cancer in mice. Nat Commun 6, 10071.

36. Bird, J.J. et al. (1998) Helper T cell differentiation is controlled by the cell cycle. Immunity 9 (2), 229-37. 
37. Leoni, C. et al. (2015) Epigenetics of T lymphocytes in health and disease. Swiss Med Wkly 145, w14191.

38. Lee, P.P. et al. (2001) A critical role for Dnmt1 and DNA methylation in T cell development, function, and survival. Immunity 15 (5), 763-74.

39. Gamper, C.J. et al. (2009) Identification of DNA methyltransferase 3a as a T cell receptor-induced regulator of Th1 and Th2 differentiation. J Immunol 183 (4), 2267-76.

40. Schoenborn, J.R. et al. (2007) Comprehensive epigenetic profiling identifies multiple distal regulatory elements directing transcription of the gene encoding interferon-gamma. Nat Immunol 8 (7), 732-42.

41. Durek, P. et al. (2016) Epigenomic Profiling of Human CD4(+) T Cells Supports a Linear Differentiation Model and Highlights Molecular Regulators of Memory Development. Immunity 45 (5), 1148-1161.

42. Komori, H.K. et al. (2015) Defining CD4 T cell memory by the epigenetic landscape of CpG DNA methylation. J Immunol 194 (4), 1565-79.

43. Ichiyama, K. et al. (2015) The methylcytosine dioxygenase Tet2 promotes DNA demethylation and activation of cytokine gene expression in T cells. Immunity 42 (4), 613-26.

44. Tsagaratou, A. et al. (2014) Dissecting the dynamic changes of 5-hydroxymethylcytosine in T-cell development and differentiation. Proc Natl Acad Sci U S A 111 (32), E3306-15.

45. Nestor, C.E. et al. (2016) 5-Hydroxymethylcytosine Remodeling Precedes Lineage Specification during Differentiation of Human CD4(+) T Cells. Cell Rep 16 (2), 559-570.

46. Ko, M. et al. (2011) Ten-Eleven-Translocation 2 (TET2) negatively regulates homeostasis and differentiation of hematopoietic stem cells in mice. Proc Natl Acad Sci U S A 108 (35), 14566-71.

47. Carty, S.A. et al. (2018) The Loss of TET2 Promotes CD8(+) T Cell Memory Differentiation. J Immunol 200 (1), 82-91.

48. Tsagaratou, A. et al. (2017) TET proteins regulate the lineage specification and TCR-mediated expansion of iNKT cells. Nat Immunol 18 (1), 45-53.

49. Yue, X. et al. (2016) Control of Foxp3 stability through modulation of TET activity. J Exp Med 213 (3), 377-97.

50. Yang, R. et al. (2015) Hydrogen Sulfide Promotes Tet1- and Tet2-Mediated Foxp3 Demethylation to Drive Regulatory T Cell Differentiation and Maintain Immune Homeostasis. Immunity 43 (2), 25163.

51. Amabile, A. et al. (2016) Inheritable Silencing of Endogenous Genes by Hit-and-Run Targeted Epigenetic Editing. Cell 167 (1), 219-232 e14.

52. Roth, T.L. et al. (2018) Reprogramming human T cell function and specificity with non-viral genome targeting. Nature 559 (7714), 405-409.

53. Seki, A. and Rutz, S. (2018) Optimized RNP transfection for highly efficient CRISPR/Cas9mediated gene knockout in primary T cells. J Exp Med 215 (3), 985-997.

54. Schafer, A. et al. (2018) Impaired DNA demethylation of C/EBP sites causes premature aging. Genes Dev 32 (11-12), 742-762. 
55. Calo, E. and Wysocka, J. (2013) Modification of enhancer chromatin: what, how, and why? Mol Cell 49 (5), 825-37.

56. $\mathrm{Wu}, \mathrm{H}$. et al. (2014) Single-base resolution analysis of active DNA demethylation using methylase-assisted bisulfite sequencing. Nat Biotechnol 32 (12), 1231-40.

57. Bachman, M. et al. (2014) 5-Hydroxymethylcytosine is a predominantly stable DNA modification. Nat Chem 6 (12), 1049-55.

58. Lister, R. et al. (2013) Global epigenomic reconfiguration during mammalian brain development. Science 341 (6146), 1237905.

59. Kim, H.S. et al. (2018) Pluripotency factors functionally premark cell-type-restricted enhancers in ES cells. Nature 556 (7702), 510-514.

60. Verma, N. et al. (2018) TET proteins safeguard bivalent promoters from de novo methylation in human embryonic stem cells. Nat Genet 50 (1), 83-95.

61. Gu, T. et al. (2018) DNMT3A and TET1 cooperate to regulate promoter epigenetic landscapes in mouse embryonic stem cells. Genome Biol 19 (1), 88.

62. Tomkova, M. and Schuster-Bockler, B. (2018) DNA Modifications: Naturally More Error Prone? Trends Genet 34 (8), 627-638.

63. Tomkova, M. et al. (2016) 5-hydroxymethylcytosine marks regions with reduced mutation frequency in human DNA. Elife 5.

64. Montagner, S. et al. (2016) TET2 Regulates Mast Cell Differentiation and Proliferation through Catalytic and Non-catalytic Activities. Cell Rep 15 (7), 1566-1579.

65. Williams, K. et al. (2011) TET1 and hydroxymethylcytosine in transcription and DNA methylation fidelity. Nature 473 (7347), 343-8.

66. Zhang, Q. et al. (2015) Tet2 is required to resolve inflammation by recruiting Hdac2 to specifically repress IL-6. Nature 525 (7569), 389-393.

67. Buscarlet, M. et al. (2017) DNMT3A and TET2 dominate clonal hematopoiesis and demonstrate benign phenotypes and different genetic predispositions. Blood 130 (6), 753-762.

68. Zang, S. et al. (2017) Mutations in 5-methylcytosine oxidase TET2 and RhoA cooperatively disrupt T cell homeostasis. J Clin Invest 127 (8), 2998-3012.

69. Ballestar, E. et al. (2006) The epigenetic face of systemic lupus erythematosus. J Immunol 176 (12), 7143-7.

70. Bock, C. et al. (2010) Quantitative comparison of genome-wide DNA methylation mapping technologies. Nat Biotechnol 28 (10), 1106-14.

71. Lentini, A. et al. (2018) A reassessment of DNA-immunoprecipitation-based genomic profiling. Nat Methods 15 (7), 499-504.

72. Yu, M. et al. (2012) Base-resolution analysis of 5-hydroxymethylcytosine in the mammalian genome. Cell 149 (6), 1368-80.

73. Booth, M.J. et al. (2012) Quantitative sequencing of 5-methylcytosine and 5hydroxymethylcytosine at single-base resolution. Science 336 (6083), 934-7. 
74. Tsagaratou, A. and Rao, A. (2013) TET proteins and 5-methylcytosine oxidation in the immune system. Cold Spring Harb Symp Quant Biol 78, 1-10.

75. Schutsky, E.K. et al. (2018) Nondestructive, base-resolution sequencing of 5hydroxymethylcytosine using a DNA deaminase. Nat Biotechnol.

76. Karemaker, I.D. and Vermeulen, M. (2018) Single-Cell DNA Methylation Profiling: Technologies and Biological Applications. Trends Biotechnol 36 (9), 952-965.

77. Stricker, S.H. et al. (2017) From profiles to function in epigenomics. Nat Rev Genet 18 (1), 51-66.

78. Liu, X.S. et al. (2016) Editing DNA Methylation in the Mammalian Genome. Cell 167 (1), 233247 e17.

79. Vojta, A. et al. (2016) Repurposing the CRISPR-Cas9 system for targeted DNA methylation. Nucleic Acids Res 44 (12), 5615-28.

80. Galonska, C. et al. (2018) Genome-wide tracking of dCas9-methyltransferase footprints. Nat Commun 9 (1), 597.

81. Liu, X.S. et al. (2018) Rescue of Fragile X Syndrome Neurons by DNA Methylation Editing of the FMR1 Gene. Cell 172 (5), 979-992 e6.

82. Greally, J.M. (2018) A user's guide to the ambiguous word 'epigenetics'. Nat Rev Mol Cell Biol 19 (4), 207-208. 
Figure 1. Genomic distribution of $\mathbf{5 m C}$. Most of the genome is highly methylated, and most of the unmethylated $\mathrm{CpGs}$ are found in $\mathrm{CpG}$ islands $[8,10]$. Methylation of proximal regulatory regions such as promoters usually leads to transcriptional silencing. Active distal regulatory regions such as enhancers also display reduced percentage of $\mathrm{CpG}$ methylation. Blue boxes indicate gene bodies (exons, 3'- and 5'-untranslated regions).

Figure 2. Dynamics of global levels of genomic $5 \mathrm{mC}$ and $5 \mathrm{hmC}$ in $\mathrm{T}$ lymphocytes. Activation of naïve $\mathrm{T}$ lymphocytes leads to extensive cell proliferation coupled with differentiation and a global reduction of both $5 \mathrm{mC}$ and $5 \mathrm{hmC}$. An increase of $5 \mathrm{hmC}$ is observed at specific enhancers and is associated with genes that acquire high expression upon cell differentiation, such as cytokine genes defining specific T cell subsets [41, 43-45]. APC: antigen-presenting cell; TCR: T cell receptor. 


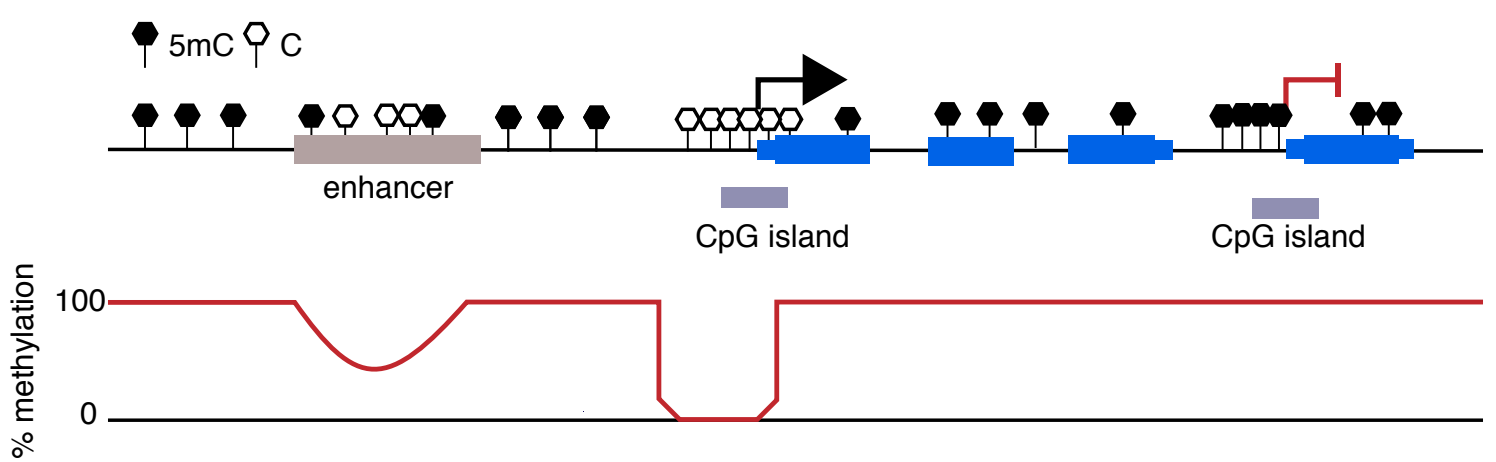




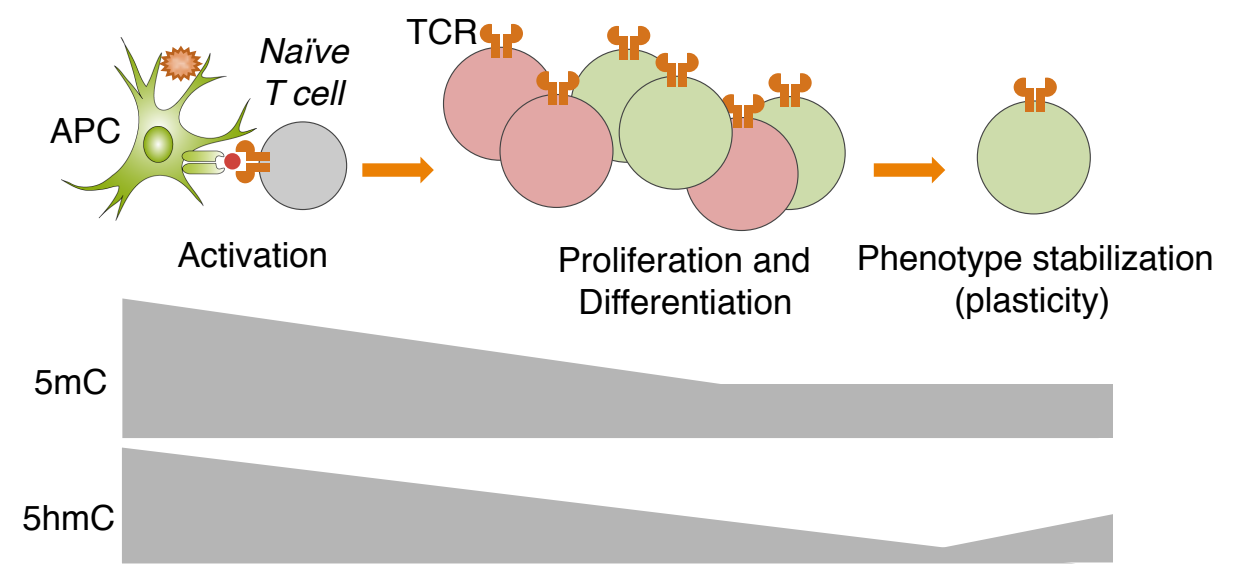

